\title{
Image Features for Image Forgery Detection System in Digital Image Forensics
}

\author{
Hlaing Htake Khaung Tin \\ Faculty of Information Science \\ University of Computer Studies, Hinthada, Myanmar \\ hlainghtakekhaungtin@gmail.com
}

\begin{abstract}
Digital image forensics is a term that is used in altered situations with slightly altered meanings. To change the meaning of an image or forensic Can be used in broadcasting to influence readers' opinions. The fake copy-move is an influence on the image; Some parts of the area are copied and copied to another district in the same area. This paper presents a study of several image features for feature extraction in digital image forensics and an investigation of many issues in image forgery detection. This paper took the exact same pixel values and presented an accurate study and evaluation of a digital image known as anesthesia for two imaging areas. A relative investigation of main methods for feature extraction is also given. Time complexity; Accuracy and detection methods based on false positives and false positives can be used to identify this type of false alarm.
\end{abstract}

Keywords: image features, forgery, detection, image forensics, image retrieval.

\section{$1 \quad$ Introduction}

In digital images, the eyes and the eyes an optical system (eg, lens), which is very similar to the human visual system such as the brain and the brain. It has an image sensor and an electronic system. Copy-move is the most widely used deletion method. This method is the first in the same image after the first copy of an image. Generally copy-move forgery aims to achieve two objectives. The test of digital imagery prevents the imitation of digital images.

Activate digital image detection method. It can be classified as a blind (passive) approach. The active approaches need advance material about the creative. The current image is supposed to be fake, and approaches try to find fake parts inside. Most active approaches require approximately advance management. For example, capturing watermarks or producing a certificate. Therefore, there is a need to remove the blind. This is where it is not necessary to rediscover previous information about source images. [1]

The way to distinguish blind images can be organized in five different courses. They are pixel-based, design basic; Camera based Physical situation based on geometry features. 
- Point or pixel-based image detection is based on notices errors near the numerical view. It can be classified, make a replica, resampling, arithmetic.

- Image-based image detection is based on the layout of the image, especially in JPEG format. JPEG Quantization, it is divided into Double JPEG and JPEG blocking. It is possible to notice the fake level Shrink image

- Camera based methods, fake simulations, identified in several stages, include chromatic aberration, color filtering and camera reply and device noise.

- Physical environment founded Fake Image Discovery, which depicts the physical environment, works on the source of the sphere. These three activities are separated into: 2D Direction Team, Light Direction 3D, and Light Environment.

- There are two types of artificial geometry based on geometry. The point is to measure the error of the objects and the substances in the domain and their location in relation to the camera. [1]

\section{$2 \quad$ Literature Review}

Digital images are a reliable way to distribute information. The quickly rising digital image and immersion software is sophisticated with advanced features. The features and operation of digital imagery can be done easily with the help of low-cost editing tools online or offline. Fake with digital imagery is an unavoidable problem, one that is often overlooked. There are two types of artificial intelligence. The major objective of the virtual appearance recognition mechanism exists to confirm the ordinal image. This is a factory that can identify misconceptions in digital images and maintain the image's integrity. Imaging of artificial imaging technology is mainly two separate approaches and they are active and passive approaches. [2]

\subsection{Active Approach}

In an active mentality, a digital image makes some pre-recorded data such as a digital mark or a digital shortcut happening the appearance when recording. However, it works against active approach during exercise. The border will be created. However, many cameras do not match the symptoms that result in the failure of an active approach.

\subsection{Maintaining the Integrity of the Specifications}

Passive or blind blind approaches enhance the focus in the field of many detectors of fake image detection, as efforts are made to unclutter the image. Verify the digital image by analyzing what is happening in the features of the image throughout this distorted processHowever, while there is a concept, there are no visible ideas that 
can be made of artificial images and images. Many existing approaches are able to identify counterfeit areas with digital imagery and discard ideas that have been discarded. In addition, the passive method is separated into numerous groups based on the complexity of the activity on digital images. Names, Types are copy, paste, drawing beside conversion.

\section{Digital Appearance Forensics}

Digital Forensics is a criminal science institute that deals with cyber crime. Digital devices are often associated with computer crimes in terms of finding and investigating items. Figure 1 illustrates the procedure for detecting fake images.

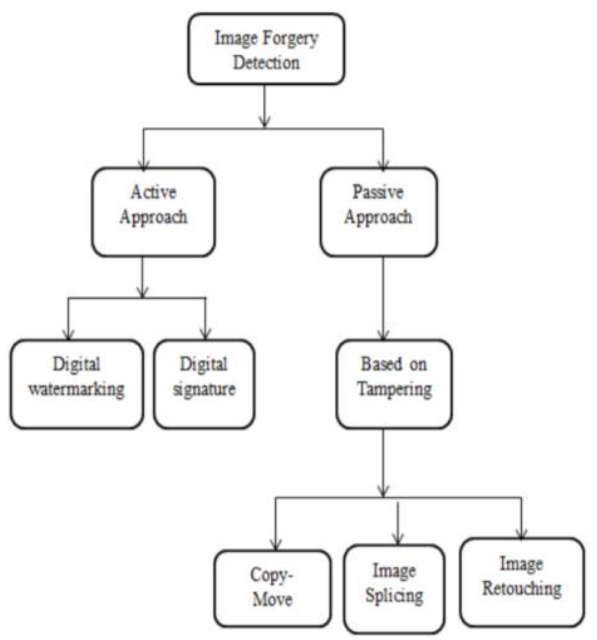

Fig.1. Arrangement of Image Fake Detection Performances [2]

Today, various forms of digital counterfeiting are being created. Image Splicing, Image Retouching and duplicate photo image splicing stands a procedure for creating an integrated image by cutting and merging two or more images. The spliced image will introduce a lot of sharp changes such as edges, edges and corners. Image Correcting refers to image correction or enhancement (color / anti / white balance) (ie, gradient correction), sharpness, noise, scars, or visible defects on the skin and material.

The most characteristic of digital attacks against cyber images is local replicas. The attacker's aim is to mislead the viewer with a deceptive image. It is also known as Copy-Move counterfeit. In this process, portion of the image is derivatived anywhere in the image, with the purpose of obscuring the necessary feature. 


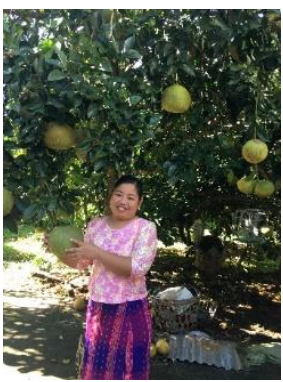

(a)

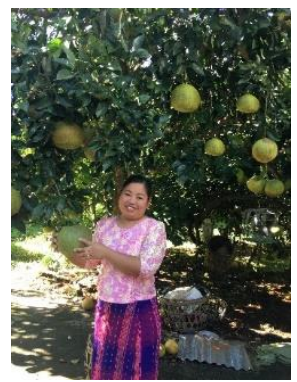

(b)

Fig. 2. The original image is figure (a) and the copy-move image is figure (b) which is copy and paste duplicate fruits.

Fake Copy-move is a widely used image editing technique that uses digital image content. It is a fake image, copying the area and hiding somewhere else in the same image structure or duplicating the object at unique times. Reducing the digital image temperature by directly copying one part of a region or digital image to another image. [3]

\section{$4 \quad$ Image Features for Image Retrieval}

Investigation of all digital forensics tools for digital forensics has been expanded. Detecting and Studying Intellectual Property Finding Fraud Electronic discovery of possible digital features; And / or authenticate documentsPhotographic features affect all aspects of an optical imaging system. Therefore, it is significant to select the right picture assets for all features of the spy routine.[2]

\subsection{RGB Color Model}

In this color format, each color stands red, green, blue mechanisms and based on Cartesian coordinate technique.The RGB's core value is the three-dimensional color space, The middle colors of cyan, magenta, yellow are bacic angles. Black is the source, white remains last location. In RGB model, the gray scale (points with the same RGB values) extends from the lines associated with this facts to white. The RGB color transformation is defined by a vector extending from the top or interior of the Cube. To simplify the process, make sure that the cube is a unit unit so that all the color values are normal. Some points of green and blue are measured at a distance of $[0,1]$. Red, green, blue appearance contains eight bit RGB image. The "full-color image" contains twenty four bit format.

\subsection{Cyan, Magenta, Yellow and Black}

An advanced color scheme usages cyan, magenta, yellow and black also called CMYK. This stands for regular color valuable printers. High-end products that include printers or printers using CMYK color patterns and accessories. The chief colors are red, green ,blue, and the main colors of pigments are magenta, magenta, 
and magenta, cyan and yellow. Converting from RGB to CMYK The values of R, $\mathrm{G}, \mathrm{B}$ can show a typical color feature from 0 to 1 . The surface of the cyan covering does not change from red to dark skin to purple to green. Consequently the four color scheme is modeled for those colors: cyan $(\mathrm{C})$, magenta $(\mathrm{M})$, yellow $(\mathrm{Y})$ and black (B).

\section{Texture Feature for Feature Extraction}

The structure is a very interesting picture. Display images from the content used for image classification. The pattern or pattern over the area of the most important feature of the texture feature is repeated and structure of that can be called textons including size and shape. The colors and tendencies can be various region. The challenge of the two structures is in the texton conversion stage and it may also be the Spatial statistics of textons in the image. Wood Carpets; Clouds Trees Land Natural properties of almost any surface such as skin and so on. It contains important information about the apparent design of the picture. The small field of the picture can be different tonal features, but surface is the main character of the area. The difference of the gray hue remain the main feature and the color box has a distinctive color in the small area of the image.

\subsection{Structural created feature mining}

Structural techniques act for a definite set of characteristics and stages of structure and tradition. In this original texture explanation needed to be original. The advantage of the composition method is to display the symbolism of the image according to the extract. However, this feature is useful for combining images rather than analysis. These procedure is not appropriate for natural features during the variation of macro and micro-textures.

\subsection{Statistical created feature mining}

Statistical created feature mining techniques are indirect to the appearance. A meaning of the undecided property which manages the connection between the gray scales of the shapes. This designs are created to compute the gray color spreading of gray standards to calculate local structures at separately opinion of the appearance and obtaining the measurements on the spreading of limited structures. The design of the statistical techniques are given one pixel as a first order and the single pixel pair known as the second order and higher (triplets or more) digits. With the exception of the spatial interaction of the pixel in the first image and it is potential to calculate the possessions of each pixel value (as a factor). The second order measurements and higher order measurements compare with each other. The culture various pixel standards taking place in a standalone state. The next greatest commonly used geometric appearance matrix recycled for surface investigation originates beginning the co-occurrence background. 


\subsection{Model created feature mining}

Evaluating process of the texture is a fractal standard and a Markov design created by the shape of the structure. It may be designed to implement surface and synthesis. This procedure defines the image as a linear combination of a possible design or a group of critical functions. The fractal designs are convenient for inventing the structural designs, as well as qualitative statistical models, as well as texture analysis and discrimination. There are methods for extracting various features based on the neighbor design and sound basis. The modification methods are the tranferring average, auto reverting, auto regressive interchange and one dimensional time sequences models. Spatial variance analysis in two-dimensional random-field models. The global random field format contains all images. Here, random fields and field models are implemented. Markov models that are likely to have a pixel intensity that is based on the intensity of the pixel in that space.

\subsection{Transform created feature mining}

In this approaches are similar method like the Fourier transform and wavelet transform, provide a description of the space in which the structure is intimately connected to the structure based on their features. The advantages of Gabor filter are usually incomplete in training because here is not any filter that normally restricts the spatial structure. These approach filters the creative image by expending and evaluating the power of the transformer picture and based on the full appearance. This is misappropriate for submissions depend on the portion of the effort. The main separation of the wavelet filters and Gabor filters are very effective for the investigation of quality.

\subsection{Shape appearance}

This remains a explanation of the object of the location, inclination and proportion of the rejection. Therefore, nature of the appearance is interpreted, translated and interpreted. There are no differences between visits and search extensions; It stays applicable after the substance of the thing is indefinite. When the appearance using as a template, require to separate the appearance to identify the area or point. It can be a challenge of shape features. The layout also divided into two classes and the first class is the edge which uses an image on the separate of an object. The next category hangs on the area in which entire objects are used. The greatest essential representations are fourier description and variations at present. The basic idea of Fourier's description is that by using the Fourier Boundary, the object is transformed into a singular form, and the transient idea be present using a mathematical instant depend onto the translation and rotation. 


\subsection{Edge and borderline appearance}

Into the edge of the appearance is a great power jump besides a high rate of hurdle from one feature to another. A pixel remains significantly alter the quality of an image. Edge detection meaningfully reduces the quantity of records and removes important material though retaining significant features of the appearance. There may be other edges around the edges, depending on the edge scale. But to some extent it is not wide. If the specified edge is set, some matters will be positioned. Simple things, such as circumference as well as shape, are simply restrained. For that reason, the boundaries are charity to estimate the borders and fragments of the view.

\subsubsection{Sobel approach}

Sobel corner recognition methods include $3 * 3$ convolution kernels. As shown in Figure 2, the kernel rotates at $90^{\circ}$. These kernels are intended to reply vertically and vertically to the height of the image compared to the feature table of the image. Kernel for vertical locations. These kernels can be used independently for the measurement of kernels. These devices can be paired to indicate the exact gradient of each point.
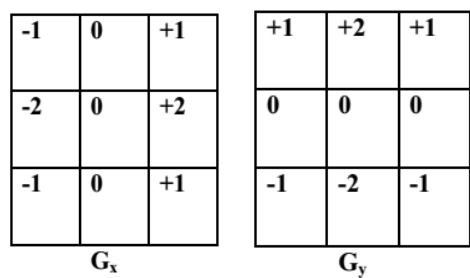

Fig.2. Masking for the Sobel operative

\subsubsection{Robert Approach}

The Robert Cross operation is fast and easy to use in 2-D spatial capabilities. Each pixel of the production characterizes the perfect scope of the spatial gradient of the appearance. The operative includes $2 \times 2$ complication kernels, as presented in Figure 3. One kernel operates the further at $90^{\circ}$. It has a lot to do through by Sobel operative.

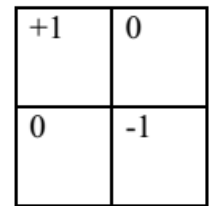

$\mathbf{G}_{\mathbf{x}}$

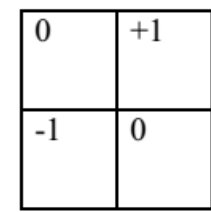

$\mathbf{G}_{\mathbf{y}}$

Fig.3. Masking for the Robert operative 


\subsubsection{Prewitt Approach}

The Prewitt operation stands associated with the Sobel operation and should be noticed in the vertical and horizontal lines of the image. Prewitt operator two parts mechanism. The vertical control is designed by Kernel Gx vertices module with the kernel Gy, as presented in Figure $4|\mathrm{Gx}|+\mid$ Gy $\mid$ offers a sign of the gradient concentration in the present pixel.
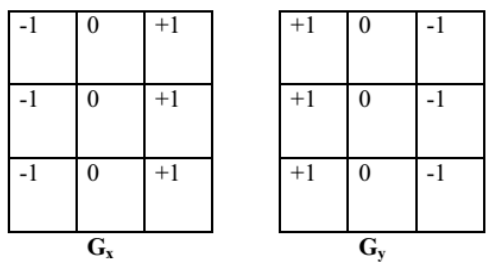

Fig.4. Masking for the Prewitt operative

\subsubsection{Canny Approach}

Canny's search procedure is extensively documented as the greatest suitable sensor. The Canny procedure customs the greatest suitable filter created on the standards. It includes reducing error rates. These include creating as many edges as possible and using edges only once. An outlet for the single extreme reaction.

Allowing Canny to receive the most possible response. The greatest strainer that encounters the three principles can be projected effectively by the firstly repetition of the Gaussian purpose. The initially phase is to smooth the image spending a Gaussian filter. Then appearance designed for the gradient of the image by giving a smooth appearance over the difficulty operation with vertical and horizontal Gaussian metaphor.

This procedure, in calculation to retaining the phase of noise reduction, also reduces the pain associated with the edge, with strong edge recognition. Finally, hysteresis is a task to avoid the band. Streaking is the failure of the lower end of the loop caused by the emergence of a disgusting operator at the bottom and bottom.

\section{Detection Accuracy}

The basic issue of this spyware that can be linked to images and simulation results. If there is no truth in the discovery of false positive information or the situation, in fact it is impossible to present the information. If there is an error in the negative data, there is an error while failing to check or indicate the test results. The system will focus only on improving the wrong features. In Copy Move detection, sometimes there is no counterfeit, but sometimes the system will detect a fake one.

False positive rate is proportional to false-positive pixels. This means that pixels are detected by a copy of the proposed non-moving surveillance algorithms. The 
false negative rate is relational to the amount of unseen features found by the algorithm.

$$
F P R=\frac{\text { Number of falsely detected pixels }}{\text { Total no: of copy moved pixels }} * 100
$$

The false negative rate is still proportional to the number of photons remaining, but is relational to the amount of unidentified pixels detected by the detection process.

$$
F N R=\frac{\text { Number of undetected copy moved pixels }}{\text { Total number of copy moved pixels }} * 100
$$

Detection accuracy is the total number of pixels acceptable by the artificial intelligence algorithm.

$D A=\frac{\text { Number of Correctly detected pixels }(\text { in copy }- \text { moved area })}{\text { Total number of copy moved pixels }} * 100$

\section{Conclusions}

This finding is a challenge in the field of digital imaging crime. Several methods are proposed to address this problem. In addition, for the better evaluation, we conducted different experiments and presented comparable results. The proposed structure combines the concept of image classification and falsification, and then replicates the images into copies. This system checks for duplicates in the input image.

\section{References}

[1] Rani Susan Oommen , "A Survey of Copy-Move Forgery Detection Techniques for Digital Images", IJIET, Vol 5, No 2, April 2015.

[2] T. Thakur, K. Singh, A. Yadav, "Blind Approach for Digital Image Forgery Detection", IJCA, Volume 179 - No.10, January 2018.

[3] V. P. Nampoothiri , N. Sugitha, 2016, "Digital Image Forgery - A threaten to Digital Forensics", IEEE (ICCPCT), 1-6.

[4] Jatin Wadhwa, Talib Ahemad "Detection of Region Duplication in Digital Images: A Digital Forensic Approach”, May 08, 2015.

[5] H. H. K. Tin, "Effective Method of Fake Currency Detection System using Image Processing”, International Journals of Advanced Research in Computer Science and Software Engineering (IJARCSSE), 2019, May, Volume -9, Issue-5. 
[6] H. H. K. Tin, "Image Features for Content Based Image Retrieval System in Image Mining", International Journals of Advanced Research in Computer Science and Software Engineering (IJARCSSE), 2019, June, Volume -9, Issue-6.

[7] H. H. K. Tin, "Comparison of the Classifiers for the Efficient Content Based Image retrieval System", International Journal of Advanced Trends in Computer Applications (IJATCA), 2019, July, Volume -6, Number-2, 27-32.

[8] H. H. K. Tin, "A Comparative Study of Classification Algorithms Using the Experimental Results in CBIR System”, International Journal of Trend in Research and Development (IJTRD), 2019, August, Volume -6, Number-4, 202-206. 